[3] H. V. Poor and S. Verdú, "Single-user detectors for multiuser channels," IEEE Trans. Commun., vol. 36, pp. 50-60, Jan. 1988.

[4] S. Verdú, "Optimum multiuser asymptotic efficiency," IEEE Trans. Commun., vol. COM-34, pp. 890-897, Sept. 1986.

[5] R. Lupas and S. Verdú, "Near-far resistance of multiuser detectors in asynchronous channels," IEEE Trans. Commun., vol. 38, pp. 496-508, Apr. 1990.

[6] J. C. Candy and G. C. Temes, Oversampling Delta-Sigma Data Converters: Theory, Design and Simulation. New York: IEEE Press, 1992.

[7] M. K. Varansi and B. Aazhang, "Optimally near-far resistant multiuser detection in differentially coherent synchronous channels," IEEE Trans. Inform. Theory, vol. 37, pp. 1006-1018, July 1991.

[8] W. C. Y. Lee, "Overview of cellular CDMA," IEEE Trans. Veh. Technol., vol. 40, pp. 291-302, May 1991.

[9] A. Kajiwara and M. Nakagawa, "Microcellular CDMA system with a linear multiuser interference canceler," IEEE J. Select. Areas Commun. vol. 12, pp. 605-611, May 1994.

[10] D. S. Chen and S. Roy, "An adaptive multiuser receiver for CDMA systems," IEEE J. Select. Areas Commun., vol. 12, pp. 808-816, June 1994.

[11] M. Honig, U. Madhow, and S. Verdú, "Blind adaptive multiuser detection," IEEE Trans. Inform. Theory, vol. 41, pp. 944-960, July 1995.

[12] R. Kohno, H. Imai, M. Hatori, and S. Pasupathy, "Combination of an adaptive array antenna and a canceller of interference for directsequence spread-spectrum multiple-access system," IEEE J. Select. Areas Commun., vol. 8, pp. 675-681, May 1990

[13] S. Verdú, "Multiuser detection," Advances in Statistical Signal Processing, Vol. 2: Signal Detection, H. V. Poor and J. B. Thomas, Ed. New York: JAI Press, 1993.

\section{Analytical Two-Dimensional IIR Notch Filter Design Using Outer Product Expansion}

\author{
Soo-Chang Pei, Wu-Sheng Lu, and Chien-Cheng Tseng
}

\begin{abstract}
This brief is concerned with the two-dimensional (2-D) digital notch filter design problem. First, the outer product expansion is used to reduce the 2-D notch filter design problem to two pairs of one-dimensional (1-D) filter design problems. Then, we develop a simple algebraic method for the design of two pairs of 1-D IIR filter design. This approach not only has closed form transfer function but also satisfies bounded input/output (BIBO) stability condition. Finally, one example is included to illustrate the proposed design method, and the 2-D IIR notch filter is applied to eliminate the sinusoidal interference superimposed on an image.
\end{abstract}

Index Terms-2-D IIR notch filter, interference removal.

\section{INTRODUCTION}

Notch filters have been an effective means for eliminating narrowband or sinusoidal interferences in certain signal processing applications ranging from power line interference cancelation for electrocardiograms to multiple sinusoidal interference removal for corrupted images. For the one-dimensional (1-D) case, several methods for the design and performance analysis of IIR and FIR notch filters have been developed, see [1]-[5] among others. For the two-

Manuscript received June 5, 1995; revised July 9, 1996. This paper was recommended by Associate Editor P. Regalia.

S.-C. Pei and C.-C. Tseng are with the Department of Electrical Engineering, National Taiwan University, Taipei, Taiwan, R.O.C.

W.-S. Lu is with the Department of Electrical and Computer Engineering, University of Victoria, Victoria, B.C., Canada V8W 3P6.

Publisher Item Identifier S 1057-7130(97)06584-1. dimensional (2-D) case, [6] and [7] proposed a method that reduces the design of a stable IIR 2-D notch filter to the design of a 2-D parallel line filter and a 2-D straight line filter. In this brief, a new approach for 2-D IIR notch filter design will be addressed.

In the IIR notch filter design, the outer product expansion of the sampled frequency response matrix of an ideal 2-D notch filter indicates that the design of a 2-D IIR notch filter can be accomplished by finding two pairs of 1-D IIR transfer functions. We develop a novel algebraic method to approximate these two type 1-D filters. This approach yields a closed-form solution with guaranteed bounded input/bounded output (BIBO) stability. Moreover, the notch filter designed exhibits a simple structure that contains only two 1-D second-order allpass filters and two 1-D first-order allpass filters. Due to the mirror image symmetry relation between the numerator and denominator of an allpass filter, the 2-D notch filter obtained can be realized by a computationally efficient lattice structure.

This brief is organized as follows. Section II presents a detailed description of the 2-D IIR notch filter design. In Section III, one example is presented to illustrate the feasibility of the proposed design technique, and the notch filter designed is then applied to an image that is corrupted with a sinusoidal interference.

\section{2-D IIR Notch FILTER DeSIGN}

The frequency response of an ideal 2-D notch filter is given by

$$
H_{d}\left(e^{j \omega_{1}}, e^{j \omega_{2}}\right)= \begin{cases}0 & \left(\omega_{1}, \omega_{2}\right)=\left(\omega_{1}^{*}, \omega_{2}^{*}\right) \text { and }\left(-\omega_{1}^{*},-\omega_{2}^{*}\right) \\ 1 & \text { otherwise }\end{cases}
$$

where $\left(\omega_{1}^{*}, \omega_{2}^{*}\right)$ is the notch frequency. Our design task is to find a stable 2-D IIR transfer function which satisfies this specification. To this end, we sample $H_{d}\left(e^{j \omega_{1}}, e^{j \omega_{2}}\right)$ and denote the sampled frequency response matrix by $\mathbf{D} \in R^{M \times N}$. If the notch frequencies $\left(\omega_{1}^{*}, \omega_{2}^{*}\right)$ and $\left(-\omega_{1}^{*},-\omega_{2}^{*}\right)$ are among the sampling grids, then the entries of matrix $\mathbf{D}$ are given by

$$
\mathbf{D}(m, n)= \begin{cases}0 & (m, n)=(k, l) \text { and }(p, q) \\ 1 & \text { otherwise } 1 \leq m \leq M, 1 \leq n \leq N\end{cases}
$$

where the $(k, l)$ th and $(p, q)$ th entries with $p=M-k+1$ and $q=N-l+1$ correspond to the notch frequencies $\left(\omega_{1}^{*}, \omega_{2}^{*}\right)$ and $\left(-\omega_{1}^{*},-\omega_{2}^{*}\right)$, respectively. Evidently, the rank of matrix $\mathbf{D}$ is three as it only contains three linearly independent columns. By using the singular value decomposition (SVD) [8], matrix $\mathbf{D}$ can be written as the following outer product expansion

$$
\mathbf{D}=\mathbf{w}_{1} \mathbf{w}_{2}^{t}-\frac{1}{2} \mathbf{u}_{1} \mathbf{u}_{2}^{t}+\frac{1}{2} \mathbf{v}_{1} \mathbf{v}_{2}^{t}
$$

where the elements of the vectors involved are given by

$$
\begin{aligned}
& \mathbf{w}_{1}(m)=1 \quad 1 \leq m \leq M \\
& \mathbf{w}_{2}(n)=1 \quad 1 \leq n \leq N \\
& \mathbf{u}_{1}(m)=\left\{\begin{array}{ll}
1 & m=k \text { and } p
\end{array} \quad 1 \leq m \leq M\right. \\
& \mathbf{u}_{2}(n)=\left\{\begin{array}{ll}
1 & n=l \text { and } q
\end{array} \quad 1 \leq n \leq N\right. \\
& \mathbf{v}_{1}(m)=\left\{\begin{array}{ll}
-j & m=k \\
j & m=p \\
0 & \text { otherwise }
\end{array} \quad 1 \leq m \leq M\right. \\
& \mathbf{v}_{2}(n)=\left\{\begin{array}{cl}
-j & n=l \\
j & n=q \\
0 & \text { otherwise }
\end{array} \quad 1 \leq n \leq N\right.
\end{aligned}
$$




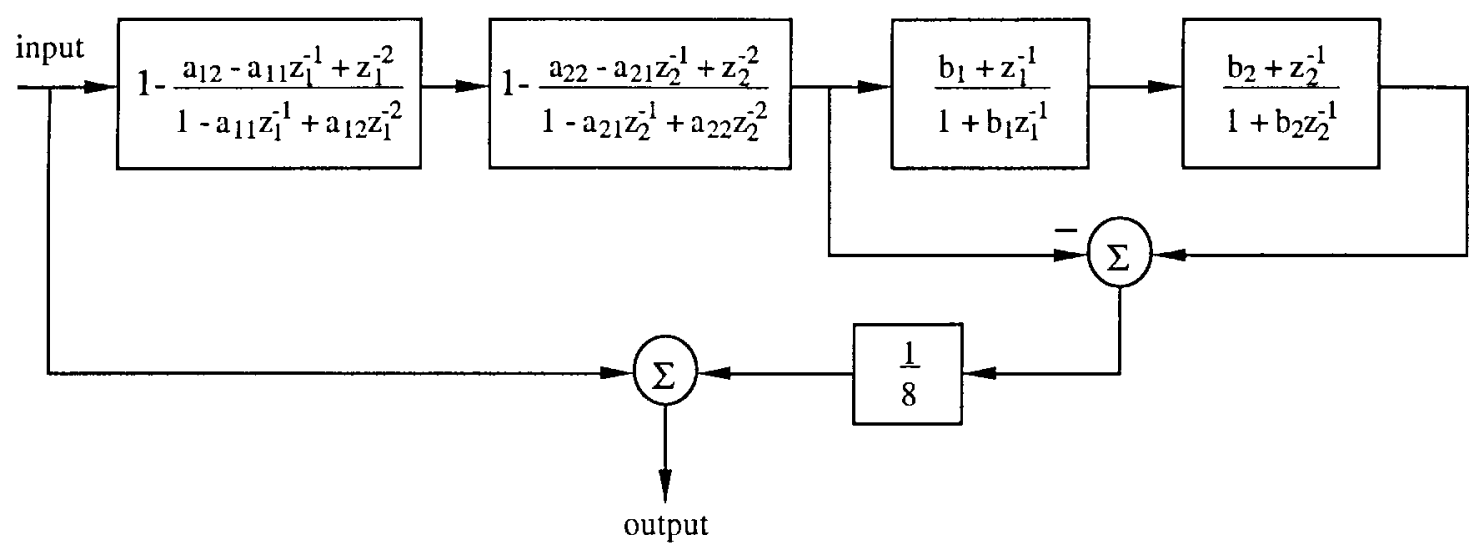

(a)

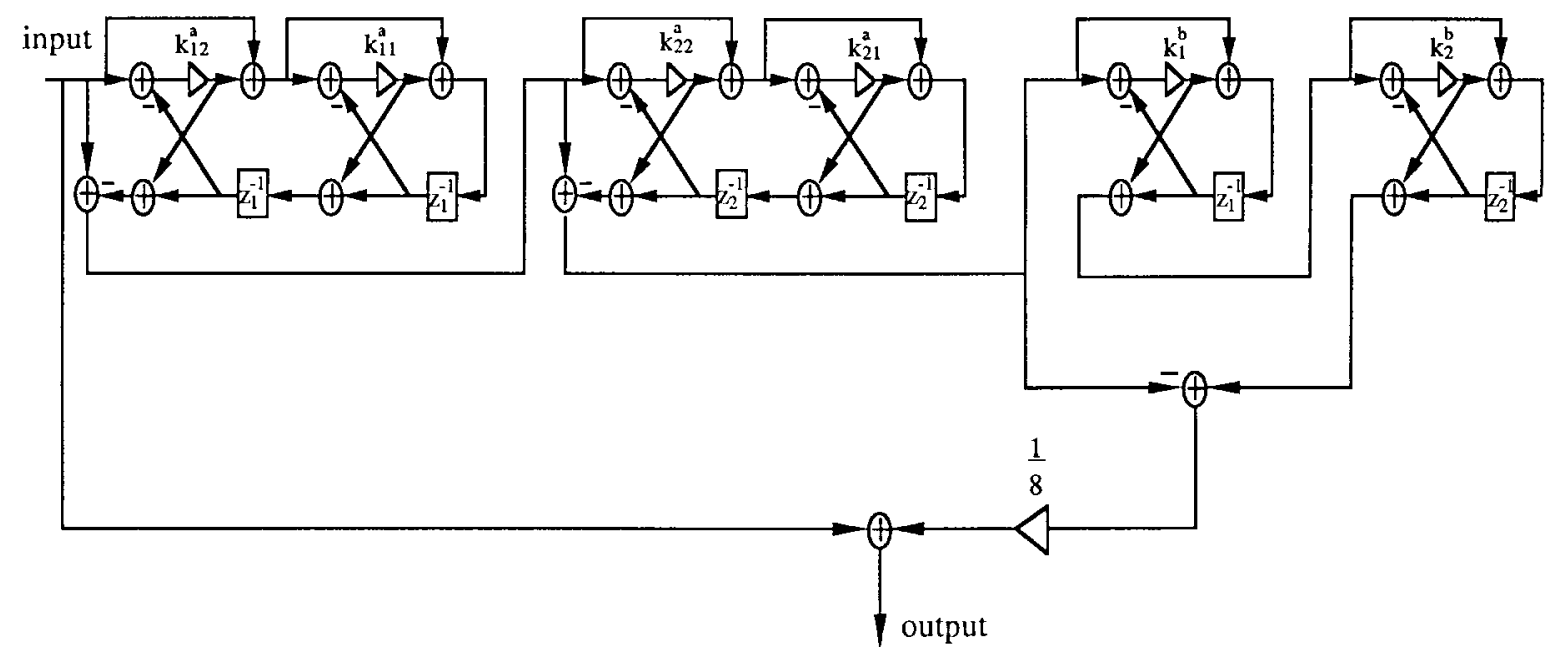

(b)

Fig. 1. 2-D IIR notch filter. (a) The block diagram of notch filter implementation. (b) The lattice form realization.

with $j=\sqrt{-1}$. This expansion suggests that the sampling frequency response $\mathbf{D}$ can be approximated by that of 1-D filters whose frequency responses approximate $\mathbf{w}_{i}, \mathbf{u}_{i}$ and $\mathbf{v}_{i}(i=1,2)$. In other words, we can state the following.

Fact 1: Given that two 1-D filters $H_{c i}\left(z_{i}\right)$ and $H_{s i}\left(z_{i}\right)$ have the following frequency responses $(i=1,2)$ :

$$
\begin{aligned}
& H_{c i}\left(e^{j \omega_{i}}\right)= \begin{cases}1 & \omega_{i}=\omega_{i}^{*} \text { and }-\omega_{i}^{*} \\
0 & \text { otherwise }\end{cases} \\
& H_{s i}\left(e^{j \omega_{i}}\right)= \begin{cases}-j & \omega_{i}=\omega_{i}^{*} \\
j & \omega_{i}=-\omega_{i}^{*} \\
0 & \text { otherwise }\end{cases}
\end{aligned}
$$

then

$$
\begin{aligned}
H_{d}\left(e^{j \omega_{1}}, e^{j \omega_{2}}\right)= & 1-\frac{1}{2} H_{c 1}\left(e^{j \omega_{1}}\right) H_{c 1}\left(e^{j \omega_{2}}\right) \\
& +\frac{1}{2} H_{s 2}\left(e^{j \omega_{1}}\right) H_{s 2}\left(e^{j \omega_{2}}\right)
\end{aligned}
$$

is the frequency response of a 2-D notch filter. (3).

Proof: This is a direct result of the outer product expansion in

From Fact 1, we see that designing a 2-D IIR notch filter can be decomposed into two types of 1-D filter design. One is the design of filter $H_{c i}\left(e^{j \omega_{i}}\right)$ defined in (5), the other is the design of filter $H_{s i}\left(e^{j \omega_{i}}\right)$ defined in $(6)(i=1,2)$. In what follows we shall address the design of these two types of filters.

\section{A. Design of Filter $H_{c i}\left(z_{i}\right)$}

The frequency response of $H_{c i}\left(e^{j \omega_{i}}\right)$ can be approximated by the second-order IIR bandpass filter whose transfer function is given by

$$
H_{b i}\left(z_{i}\right)=\frac{1}{2}\left(1-\frac{a_{i 2}-a_{i 1} z_{i}^{-1}+z_{i}^{-2}}{1-a_{i 1} z_{i}^{-1}+a_{i 2} z_{i}^{-2}}\right) \quad i=1,2
$$

where, from the results in [6], the coefficients $a_{i 1}$ and $a_{i 2}$ are given by

$$
\begin{aligned}
& a_{i 1}=\frac{2 \cos \left(\omega_{i}^{*}\right)}{1+\tan \left(\frac{\mathrm{BW}}{2}\right)} \\
& a_{i 2}=\frac{1-\tan \left(\frac{\mathrm{BW}}{2}\right)}{1+\tan \left(\frac{\mathrm{BW}}{2}\right)}
\end{aligned}
$$

with $\omega_{i}^{*}$ is the center frequency of $H_{b i}\left(z_{i}\right)$ and $\mathrm{BW}$ is the $3 \mathrm{~dB}$ bandwidth of $H_{b i}\left(z_{i}\right)$. Note that $H_{b i}\left(e^{j \omega_{i}^{*}}\right)$ is exactly equal to unity, i.e., $H_{b i}\left(e^{j \omega_{i}}\right)$ has unit gain and zero phase at $\omega_{i}=\omega_{i}^{*}$. Thus, $H_{b i}\left(e^{j \omega_{i}}\right)$ will be an excellent approximation of $H_{c i}\left(e^{j \omega_{i}}\right)$ provided that $\mathrm{BW}$ is sufficiently small.

\section{B. Design of Filter $H_{s i}\left(z_{i}\right)$}

It can readily be verified that the filter $H_{s i}\left(z_{i}\right)$ can be obtained as $H_{s i}\left(z_{i}\right)=H_{c i}\left(z_{i}\right) H_{a i}\left(z_{i}\right)$ where the frequency response of $H_{a i}\left(z_{i}\right)$ is given by

$$
H_{a i}\left(e^{j \omega_{i}}\right)= \begin{cases}-j & \omega_{i}=\omega_{i}^{*} \\ j & \omega_{i}=-\omega_{i}^{*} \\ \text { don't care } & \text { otherwise }\end{cases}
$$


Since $H_{c i}\left(z_{i}\right)$ has been designed in the preceding subsection, we now only need to design filter $H_{a i}\left(z_{i}\right)$. For simplicity, we choose $H_{a i}\left(z_{i}\right)$ to be the following first-order allpass filter

$$
H_{a i}\left(z_{i}\right)=\frac{b_{i}+z_{i}^{-1}}{1+b_{i} z_{i}^{-1}} \quad i=1,2 .
$$

Since $\left|H_{a i}\left(e^{j \omega_{i}}\right)\right|$ is equal to unity for all frequencies, i.e., $H_{a i}\left(e^{j \omega_{i}}\right)$ can be written as

$$
H_{a i}\left(e^{j \omega_{i}}\right)=e^{j \theta_{i}\left(\omega_{i}\right)}
$$

where the phase response $\theta_{i}\left(\omega_{i}\right)$ is given by

$$
\theta_{i}\left(\omega_{i}\right)=-\omega_{i}+2 \arctan \left(\frac{b_{i} \sin \left(\omega_{i}\right)}{1+b_{i} \cos \left(\omega_{i}\right)}\right) .
$$

For a stable allpass filter $H_{a i}\left(z_{i}\right)$, we have three following properties: (1) $\theta_{i}(0)=0$, (2) $\theta_{i}(\pi)=-\pi$, and (3) $\theta_{i}\left(\omega_{i}\right)$ decreases monotonically with frequency $\omega_{i}$ [9]. These properties imply that when $\omega_{i}$ goes from 0 to $\pi$ radians, the phase $\theta_{i}\left(\omega_{i}\right)$ goes from 0 to $-\pi$. Moreover, the specification in (10) implies that

$$
\theta_{i}\left(\omega_{i}^{*}\right)=-\frac{\pi}{2}
$$

That is, when $\omega_{i}=\omega_{i}^{*}$, the phase $\theta_{i}\left(\omega_{i}\right)$ reaches the point $\frac{-\pi}{2}$. Substituting (14) into (13), we obtain

$$
b_{i}=\frac{\sin \left(\frac{\omega_{i}^{*}}{2}-\frac{\pi}{4}\right)}{\sin \left(\frac{\omega_{i}^{*}}{2}+\frac{\pi}{4}\right)}
$$

Hence, the transfer function $H_{s i}\left(z_{i}\right)$ is given by

$$
H_{s i}\left(z_{i}\right)=\frac{1}{2}\left(1-\frac{a_{i 2}-a_{i 1} z_{i}^{-1}+z_{i}^{-2}}{1-a_{i 1} z_{i}^{-1}+a_{i 2} z_{i}^{-2}}\right) \frac{b_{i}+z_{i}^{-1}}{1+b_{i} z_{i}^{-1}} \quad i=1,2
$$

where the coefficients $a_{i 1}, a_{i 2}$, and $b_{i}$ are determined by (9) and (15).

\section{Design Procedure and Implementation}

Based on the above discussion, a complete procedure for the design of 2-D IIR notch filter can be summarized as follows:

Step 1: Specify notch frequency $\left(\omega_{1}^{*}, \omega_{2}^{*}\right)$ and bandwidth BW.

Step 2: Use (9) to compute filter coefficients $a_{i 1}, a_{i 2}(i=1,2)$. Construct transfer function

$$
H_{b i}\left(z_{i}\right)=\frac{1}{2}\left(1-\frac{a_{i 2}-a_{i 1} z_{i}^{-1}+z_{i}^{-2}}{1-a_{i 1} z_{i}^{-1}+a_{i 2} z_{i}^{-2}}\right) \quad i=1,2 .
$$

Step 3: Use (15) to calculate coefficients $b_{i}(i=1,2)$. Construct transfer function

$$
H_{a i}\left(z_{i}\right)=\frac{b_{i}+z_{i}^{-1}}{1+b_{i} z_{i}^{-1}} \quad i=1,2 .
$$

Step 4: Form the transfer function of the 2-D IIR notch filter as

$$
H\left(z_{1}, z_{2}\right)=1-\frac{1}{2} H_{b 1}\left(z_{1}\right) H_{b 2}\left(z_{2}\right)\left(1-H_{a 1}\left(z_{1}\right) H_{a 2}\left(z_{2}\right)\right) .
$$

Fig. 1(a) shows an implementation block diagram of the proposed 2-D notch filter. This implementation only contains two 1-D secondorder allpass filters and two 1-D first-order allpass filters. Due

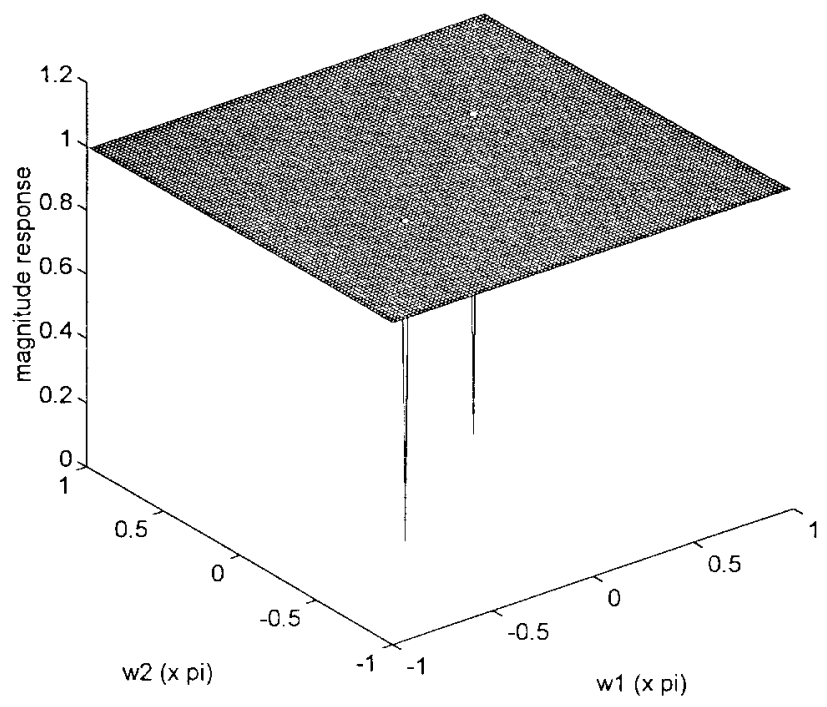

Fig. 2. The magnitude response of 2-D IIR notch filter.

to the mirror-image symmetry relation between the numerator and denominator polynomials of allpass filter, each allpass filter can be realized by the computationally efficient lattice structure with minimum multipliers and delays described in [10], as depicted in Fig. 1(b). When the filter coefficients of the direct-form allpass filter are obtained using the proposed design procedure, the lattice filter coefficients can be obtained by the transform method described in [11]. It is obvious that this lattice realization only requires seven real multiplications for computing each output sample. Moreover, the frequency response of a notch filter is very insensitive with respective to the lattice filter coefficients, and an IIR notch filter is stable if the magnitude of lattice coefficients are all smaller than unity.

\section{Design Example AND APPLICATION}

In this section, one design example of 2-D IIR notch filter is first illustrated. Next, we use the 2-D IIR notch filter to remove sinusoidal interferences superimposed on an image.

\section{Example 1: 2-D IIR Notch Filter Design}

In this example, the procedure described in Section II-C is used to design a 2-D IIR notch filter with notch frequency $\left(\omega_{1}^{*}, \omega_{2}^{*}\right)=$ $(0.4 \pi, 0.3 \pi)$. The design parameter BW is chosen as $0.001 \pi$. After some numerical computations, we obtain

$$
\begin{aligned}
a_{11} & =0.6171 \quad a_{12}=0.9969 \\
a_{21} & =1.1737 \quad a_{22}=0.9969 \\
b_{1} & =-0.1584 \quad b_{2}=-0.3294 .
\end{aligned}
$$

Fig. 2 shows the resultant magnitude response in linear scale. It is clear that the specification is well satisfied. In fact, when the bandwidth BW approaches zero, the designed notch filter will become an ideal one. Moreover, we use the transform method developed in [11] to compute the lattice filter coefficients of implementation structure in Fig. 1(b) as follows:

$$
\begin{aligned}
k_{11}^{a}=-0.3090 & k_{12}^{a}=0.9969 \\
k_{21}^{a}=-0.5878 & k_{22}^{a}=0.9969 \\
k_{1}^{b}=-0.1584 & k_{2}^{b}=-0.3294 .
\end{aligned}
$$

Since the magnitude of the lattice filter coefficients are all smaller than unity, the designed filter is stable. 


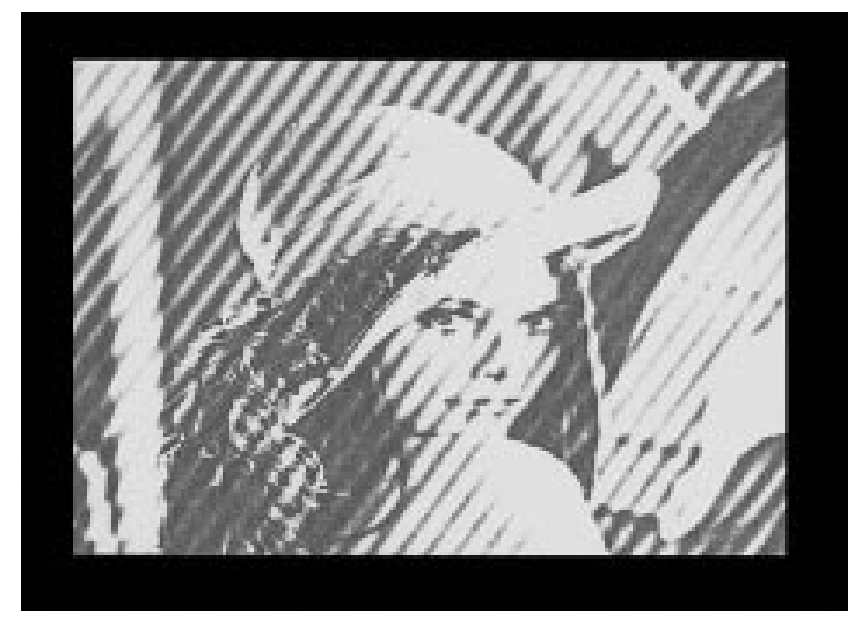

(a)

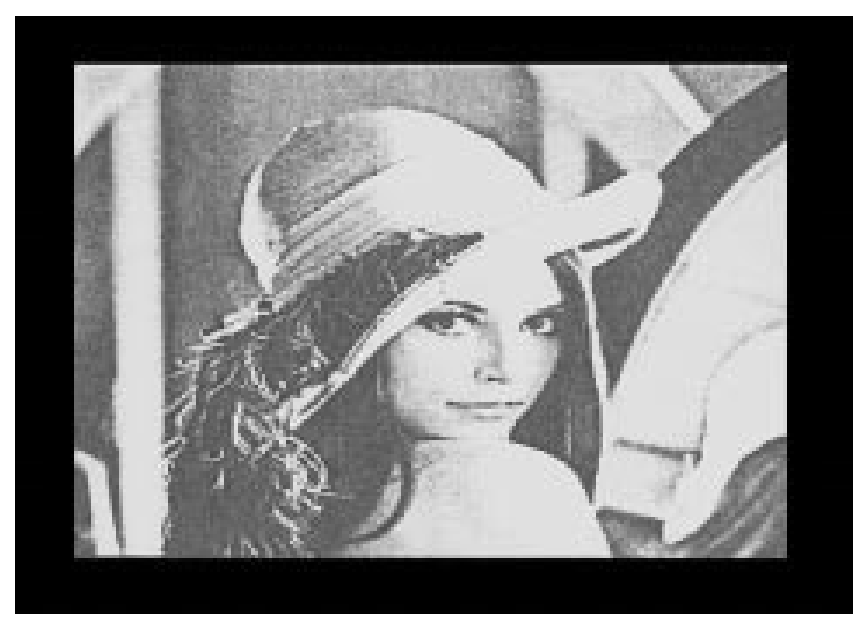

(b)

Fig. 3. Example of single sinusoidal interference removal. (a) Corrupted image. (b) Image restored by using 2-D IIR notch filter with transient suppression.

\section{Example 2: Sinusoidal Interference Removal}

In the example, we will use the 2-D IIR notch filter to remove single sinusoidal interference superimposed on an image. The scenario of experiment is the same as the one described in [6] except that the image size here is $256 \times 256$. The image shown in Fig. 3(a) is the Lena image corrupted by a sinusoidal pattern of the form

$$
30 \sin (0.1 \pi m+0.2 \pi n) \text {. }
$$

Now, we design a 2-D IIR notch filter with $\left(\omega_{1}^{*}, \omega_{2}^{*}\right)=(0.1 \pi, 0.2 \pi)$ and $\mathrm{BW}=0.01 \pi$ to remove the interference in spatial domain. In order to eliminate the transient states degradation due to incomplete boundary data, the transient state suppression algorithm developed in [12] is adopted here. The filtered image, shown in Fig. 3(b), is clearly free from interference. Moreover, for an image with size $N \times N$, the IIR notch filter implemented with the lattice structure in Fig. 1(b) only requires $7 \mathrm{~N}^{2}$ real multiplications to restore an image, but the IIR notch filter in [6] requires $20 \mathrm{~N}^{2}$ real multiplications. Hence, the notch filtering technique proposed in this brief is more efficient than the one in [6].

\section{CONCLUSION}

In this brief, 2-D IIR notch filter design problem has been investigated. First, the outer product expansion is used to reduce the 2-D notch filter design problem to two pairs of 1-D filter design problems. Then, we provide the closed-form solutions for the design of two pairs of 1-D IIR filters. Finally, the design and application examples are presented to demonstrate the performance of the proposed design methods. However, only fixed notch filters are considered here. Thus, it is interesting to develop a 2-D adaptive notch filtering algorithm. This topic will be studied in the future.

\section{REFERENCES}

[1] R. Carney, "Design of a digital notch filter with tracking requirements," IEEE Trans. Space Electron. Telem., vol. SET-9, pp. 109-114, Dec. 1963.

[2] K. Hirano, S. Nishimura, and S. K. Mitra, "Design of digital notch filters," IEEE Trans. Circuits Syst., vol. CAS-21, pp. 540-546, July 1974.

[3] M. H. Er, "Designing notch filter with controlled null width," Signal Processing, vol. 24, no. 3, pp. 319-329, Sept. 1991.

[4] G. W. Medlin, "A novel design technique for tuneable notch filters," in Proc. Int. Symp. Circuits Syst., New Orleans, LA, May 1990, pp. 471-474.

[5] S. C. Dutta Roy, S. B. Jain, and B. Kumar, "Design of digital FIR notch filters," Proc. Inst. Elect. Eng.-Vis. Image Signal Processing, vol. 141, pp. 334-338, Oct. 1994.

[6] S. C. Pei and C. C. Tseng, "Two dimensional IIR digital notch filter design," IEEE Trans. Circuits Syst. II, vol.-41, pp. 227-231, Mar. 1994.

[7] _ , "Corrections to two dimensional IIR digital notch filter design," IEEE Trans. Circuits Syst. II, vol. 41, p. 630, Sept. 1994.

[8] E. H. Golub and C. Van Loan, Matrix Computations. Baltimore, MD: Johns Hopkins Univ. Press, 1983

[9] X. Zhang and H. Iwakura, "Novel method for designing digital allpass filters based on eigenvalue problem," Electron. Lett., vol. 29, no. 14, pp. 1279-1281, July 1993.

[10] P. A. Regalia, S. K. Mitra, and P. P. Vaidyanathan, "The digital all-pass filter: A versatile signal processing building block," Proc. IEEE, vol. 76, pp. 19-37, Jan. 1988.

[11] A. H. Gray Jr. and J. D. Markel, "Digital lattice and ladder filter synthesis," IEEE Trans. Audio Electroacoust., vol. AU-21, pp. 491-500, Dec. 1973

[12] W. E. Alexander, "Initial condition transient suppression for 2dimensional recursive filters," in Proc. IEEE Int. Conf. Acoust. Speech, Signal Processing, 1984, pp. 2011-2014. 